Delft University of Technology Software Engineering Research Group Technical Report Series

\title{
Visualizing Testsuites to Aid in Software Understanding
}

Bas Cornelissen, Arie van Deursen, Leon Moonen and Andy Zaidman

Report TUD-SERG-2006-003

T̛UDelft

SERG 
TUD-SERG-2006-003

Published, produced and distributed by:

Software Engineering Research Group

Department of Software Technology

Faculty of Electrical Engineering, Mathematics and Computer Science

Delft University of Technology

Mekelweg 4

2628 CD Delft

The Netherlands

ISSN 1872-5392

Software Engineering Research Group Technical Reports:

http://www.se.ewi.tudelft.nl/techreports/

For more information about the Software Engineering Research Group:

http://www.se.ewi.tudelft.nl/

(C) copyright 2006, Software Engineering Research Group, Department of Software Technology, Faculty of Electrical Engineering, Mathematics and Computer Science, Delft University of Technology. All rights reserved. No part of this series may be reproduced in any form or by any means without prior written permission of the publisher. 


\title{
Visualizing Testsuites to Aid in Software Understanding
}

\author{
Bas Cornelissen ${ }^{1}$, Arie van Deursen ${ }^{2}$, Leon Moonen ${ }^{2}$, and Andy Zaidman ${ }^{1}$ \\ ${ }^{1}$ Delft University of Technology - s.g.m.cornelissen@tudelft.nl, a.e.zaidman@tudelft.nl \\ ${ }^{2}$ Delft University of Technology and CWI - Arie.vanDeursen@tudelft.nl, Leon.Moonen@computer.org
}

\begin{abstract}
Agile software development methods such as eXtreme Programming have brought renewed attention to testing during the software development process, both as a quality assurance method and as a form of live documentation. It is for this reason that a software system's testsuite is an ideal starting point for gaining knowledge about its inner workings. In this paper, we propose to use sequence diagrams to visualize information that is dynamically obtained from testsuites. We employ abstraction techniques such as constructor hiding and stack depth limitation to make the diagrams more scalable. We use JPACMAN as a case study to validate our results by consulting with domain experts, and use their feedback to fine-tune our techniques.
\end{abstract}

\section{Introduction}

When implementing and maintaining software systems, testing is of vital importance. The advent of Agile software development methods such as eXtreme Programming (XP) [4], Scrum [28] and DSDM [30] has ensured that testing is given much attention. In these processes, testing serves two distinct purposes. Not only is testing considered to be essential when implementing and maintaining software to help increase the quality and correctness of code, but (unit) tests are also a means of documentation $[10,18,19,9,11]$. Test-driven development [5], which is related to Agile software development, implies creating and maintaining an extensive testsuite in order to guarantee that the various components work correctly, both individually (by means of unit tests) and as a whole (through use of acceptance tests).

Various testing methodologies have been devised for a broad range of programming languages. Among these approaches are the well-known $x$ Unit frameworks that were originally based on the JUNIT design by Beck and Gamma [6]. JUNIT allows for the specification of both unit tests and acceptance tests, and is relatively easy to use. A JUNIT testcase consists of several steps: the creation of a fixture, exercising the method(s) under test, comparing the results (i.e., assertions), and the teardown. Typically, a set of testcases is run as a testsuite.

Visualizing testsuites Although most testcases might seem easily comprehensible at first sight, they quickly tend to be- come relatively complex for someone who wants to have a look "under the hood". When considering object-oriented systems, typically there are lots of interactions taking place between many objects, thus hindering comprehensibility. Therefore, we propose to visualize testcases in a way that is sufficiently detailed for comprehension purposes and, at the same time, remains human readable. As such, the general approach we follow can be described as either analyzing or tracing the testcases, applying certain abstractions and, finally, presenting the results. Such visualizations can be extremely helpful in the context of Agile software development, in which tests serve as documentation.

UML sequence diagrams $[20,27]$ are a useful means to visualize a system's behavior [22]. A scenario diagram is a somewhat simplified version of a sequence diagram that describes a single scenario, i.e., depicting one particular flow of control. Scenario diagrams provide detailed information on interactions at either the class level or the object level, and are easy to read because the chronological order is intuitive. However, if no abstractions are applied, scenario diagrams tend to become too large: the complete execution of a sizeable software system would result in a scenario diagram that contains more information than the reader can handle [23].

Goal In general terms we want to gain more knowledge about the system at hand, while the ultimate goal is to facilitate the implementation of change requests. We propose that users build up this knowledge by studying reconstructed scenario diagrams from JUNIT testsuites. Our choice for using testcases as scenarios is inspired by three major factors: (1) XP and Agile software development in general advocate the use of tests as a form of documentation [18, 19, 9, 11], (2) finding relevant scenarios for executing software and performing dynamic analysis on them (e.g., constructing visualizations) is not straightforward when domain knowledge is lacking [33], and (3) scalability problems can in part be overcome by the careful selection of relatively concise execution scenarios, i.e., testcases. This also fits in the as-needed reverse engineering strategy that is often advocated in dynamic analysis [33].

Research Questions To structure our investigation towards these goals, we define the following research questions:

- Are tests as typically written in JUNIT a suitable starting point for program comprehension?

- Do scenario diagrams obtained from these tests offer in- 
sight in the way the application works?

- Which abstractions do we need to make these diagrams more easily understandable?

- JUNIT can be used to write tests focused on one class or method (which are true unit tests), as well as for creating high level testcases which act more like system or acceptance tests. Should these be treated differently when leveraging testsuites for program comprehension?

In order to address these questions and to validate our techniques, we conduct an extensive case study on JPACMAN, a game written for educational purposes, that is a very suitable case since it features a large testsuite. By consulting the developers we aim to collect useful feedback, particularly with regard to (generic) abstractions that are needed in this context.

This paper is structured as follows. The next section outlines the issues and design choices. Section 3 presents our Scenario Diagram Reconstruction (SDR) framework. Section 4 discusses the prototype implementation that was used for the case study in Section 5. Related work is described in Section 6, and we conclude with Section 7.

\section{Design Options and Requirements}

In the course of converting testsuites to scenario diagrams, we face several challenges. This section addresses the most prominent issues and requirements.

\subsection{Design Options}

Static vs. dynamic In obtaining scenario diagrams from testcases, we can choose whether to capture the system's behavior by means of static analysis (i.e., analyzing the code) or through dynamic analysis (i.e., tracing the execution). The well-known benefits of a static approach are the genericity and compactness, whereas a dynamic technique potentially offers more details on important aspects such as late binding.

A frequently mentioned drawback of dynamic analysis is that one needs specific scenarios to exercise the system. These scenarios, however, come for free when using testcases to drive the comprehension process. Another drawback of dynamic analysis is that the information derived represents only part of the system's behavior. We take the position that in our context, having more detailed information pertaining directly to testcases is to be favored over genericity.

Recognizing stages in a scenario The second issue that arises is how one can distinguish between particular stages during the execution of a scenario. In particular, we are interested in recognizing the various stages in executing a testcase, i.e., the test initialization, test execution, result validation and teardown ${ }^{1}$. Being able to separate these stages provides a more focused view on the execution of a testcase and allows the viewer to skip a stage (if desired), or to put it in a separate

\footnotetext{
${ }^{1}$ The earlier naming conventions for these stages were replaced by annotations in JUNIT v4.
}

diagram. Furthermore, as we will see later, recognizing which methods and objects play a role in the various stages allows for particular filtering and abstraction techniques. For example, the assertions made during the result validation stage of a (unit) test are generally of less interest in the context of comprehending the inner workings of a software system.

Class vs. object level Another design choice concerns whether we want to trace the interactions at the class level or at the object level. The former is easier because it does not require an administration of all runtime objects, whereas being able to distinguish between objects provides detailed information on object interactions, and exposes occurrences of polymorphism and late binding, which is very suitable for display in scenario diagrams. However, with diagrams at the class level already being susceptible to size problems, one will definitely encounter scalability issues with additional object information.

Scalability Despite the fact that (in general) the execution of a unit test is relatively short, scalability problems are inevitable. Most simple unit tests will probably fit on a single or a limited number of "screens", but more complex testcases (i.e., acceptance tests) induce too many interactions to simply put in a diagram without applying any form of abstraction. Therefore, we will need abstraction and filtering techniques that are both efficient and useful: we must determine which interactions are presumably irrelevant and can therefore be combined or omitted for an initial view.

\subsection{Design Requirements}

In order to create a flexible and generic tool that can aid in the understanding of a variety of software systems, we define the following requirements:

Obliviousness The system-under-analysis should not require any changes to its implementation to enable its examination.

Extensibility The framework should allow addition of new or customized (1) event listeners to capture (dynamic) system behavior, (2) abstraction and filtering techniques to combine or omit information, and (3) output renderings to facilitate flexible visualization and embedding in other environments (e.g., a software development environment like Eclipse).

Interactivity The framework should allow interactive exploration of reconstructed diagrams. We take the position that a human in the loop is required since no automated analysis can capture which improvements in comprehensibility are achieved by employing certain abstractions or filterings. The user should remain in control of the level of detail.

Comprehensibility When talking about generating "understandable" sequence diagrams, we need criteria that capture comprehensibility. The following two properties, taken from the realm of visual programming languages, seem appropriate to determine when a diagram is understandable [32]: (1) Accessibility of related information. Viewing related information in close proximity is essential. When two screen objects are not in close proximity, there is the psychological claim 


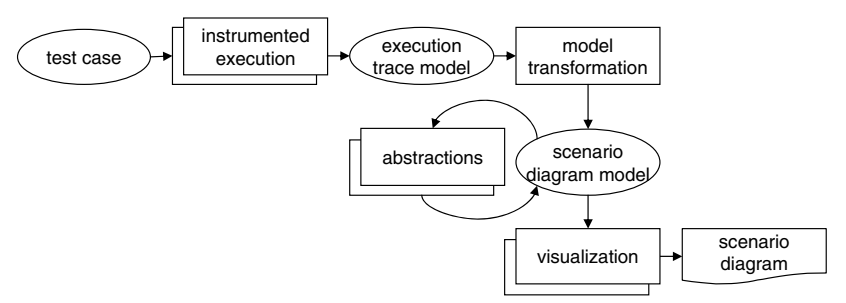

Figure 1. Overview of the SDR framework.

that these objects are not tightly related to each other or are not dedicated to solving the same problem; (2) Use of screen real estate. The term "screen real estate" refers to the size of a physical display screen and connotes the fact that screen space is a valuable resource. Making optimal use of the available screen real estate is necessary to save the user time when locating information.

\section{SDR Framework}

Based on the design options and issues discussed earlier, we have designed a framework for Scenario Diagram Reconstruction (SDR). Figure 1 provides an overview. It is an extensible framework in that there is a clear separation between the tracing part, the abstraction part and the rendering of scenario diagrams. In the remainder of this section we will discuss and motivate various features of the framework.

\subsection{Tracing testcases}

There exist several methods to obtain traces from software systems, among which the most commonly used are manually instrumenting code (e.g., [2]), using a debugger or profiler [34], and instrumentation through aspects [1]. The advantages and shortcomings of each of these techniques are mostly well-known (and, for example, discussed in [33]).

Our framework uses AOP (Aspect Oriented Programming) since aspects offer a very flexible solution in this context. Moreover, using AOP satisfies our requirement of obliviousness: the system-under-analysis does not require any changes to enable its examination. Aspects allow us to specify very accurately which parts of the execution are to be considered, where tracing must start and stop, and what postprocessing needs to take place. In addition, aspects can obtain detailed information on all interactions, such as the unique objects that are involved, the runtime arguments, and the actual return values in case of method and constructor calls.

Finally, most aspect languages allow for patterns in the definition of which classes and interactions are to be traced (i.e., pointcuts). This enables us to distinguish between the various stages in a testcase by exploiting the naming conventions or annotations used for these stages within xUnit frameworks.

Our traces are captured in a common format describing the events that can take place during the execution of a system.

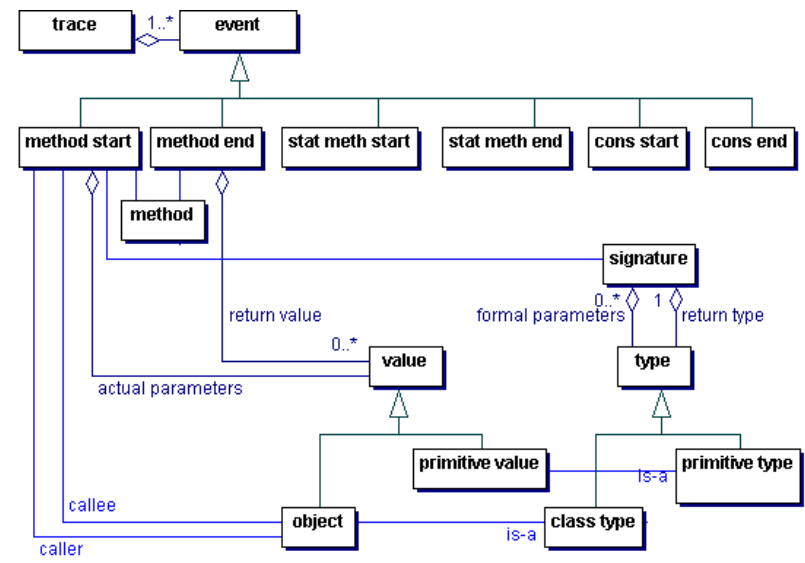

Figure 2. Trace metamodel.

We make a distinction between the beginnings and endings of method calls, static method calls and constructor calls. An execution trace consists of a set of events, each type of event having its own characteristics. Associated with each method start, for example, is a method identifier, two objects (i.e., the caller and the callee), a signature, and zero or more (runtime) parameters; A method end-event features a method identifier and the actual return value.

Figure 2 shows the model describing our traces. Note that due to space constraints, details for static method calls and constructor calls were omitted, but these are similar to those of the regular calls that are shown in the figure.

\subsection{Abstraction and Filtering}

In order to make large scenario diagrams easier to read, we need several abstraction and filtering techniques to reduce the amount of information. In the context of scenario diagrams, one intuitively thinks of omitting or joining objects, classes or combining interactions to shrink the dimensions of the diagram. But which messages and objects can be removed or combined while ensuring that the essence of the testcase is preserved? Below, we collect and discuss a catalog of abstractions and filterings that we have defined in the context of test-scenario diagrams.

- Constructor hiding. Omit all constructors and their control flows. This is especially applicable in the initialization stages of complex testcases.

- Selective constructor hiding. Omit irrelevant constructors, i.e., those pertaining to objects that are never used. This allows reduction of the diagram's dimensions without loss of essential information. The selection in one particular stage can also be based on objects that appear in certain other stages of the scenario. This can, for example, be used to filter objects created for result validation in testcases.

- Maximum stack depth. Omit all interactions that occur above a certain stack depth threshold. Intuitively, this filters low level messages that tend to be too detailed, at 


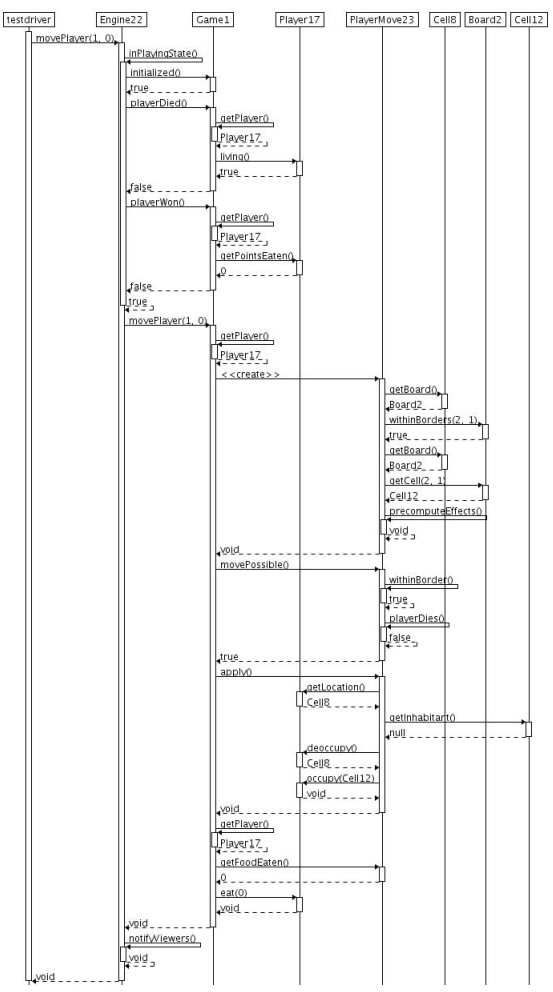

Figure 3. Reconstructed scenario diagram at a low abstraction level.

least for an initial viewing. This is illustrated by Figures 3 and 4. A similar abstraction was applied in [25], in which (in a static context) the length of a call chain is considered.

- Minimum stack depth. Omit all interactions below a certain threshold, i.e., remove high-level interactions. This can be used to omit "control messages" that start up a certain scenario.

- Fragment selection by using stack depths. Choose a set of methods of interest and, by selecting appropriate minimum and maximum stack depths, highlight its direct environment. Method selection can e.g. be done using the techniques described by Zaidman et al. [34].

- Fragment selection by zooming. Zoom in on areas of interest [29].

- Pattern recognition. Summarize recurrent patterns [22, 31]. Patterns may be identical or very similar (sets of) method calls.

- Object merging (clustering). Merge lifelines of closely related (or manually selected) objects, thereby hiding their mutual interactions [24].

- Colors. Use color techniques (e.g., graying out, or using different colors for various fragments) to "distinguish" between (ir-)relevant parts [29].

- Getters and setters. Omit simple getters and setters. We will see this abstraction applied in the case study.

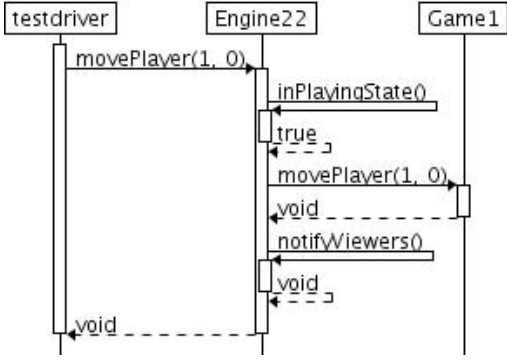

Figure 4. Reconstructed scenario diagram at a high abstraction level.

- Textual techniques. (1) Omit return values in case of void methods, (2) abbreviate full parameters, and/or (3) abbreviate return values.

\section{Implementation}

The design mentioned in the previous section has been implemented in a prototype tool, which we call the Sequence Diagram Reconstruction (SDR) tool. It is entirely implemented in Java and AspectJ and was used to conduct the case studies described later in the paper.

Scenario diagram model The scenario diagram metamodel that we use is loosely based on the UML standard for sequence diagrams [20]. It is a simplified version in that we do not focus on genericity: a scenario diagram corresponds with the execution of one particular scenario (one testcase). As such, constructs such as conditions and repetitions are omitted in our model, whereas detailed information such as runtime parameters and actual return values are included in each diagram. A distinction is made between various types of events.

The model is depicted in Figure 5, and illustrates that we define a scenario diagram to be a sequence of messages between objects. Each message is associated with a corresponding method, has zero or more actual parameters and possibly a return value.

Tracing Tracers are implemented by specializing the abstract tracing aspect Abstract Tracer that specifies the common (default) behavior. Upon initialization, it starts an event listener

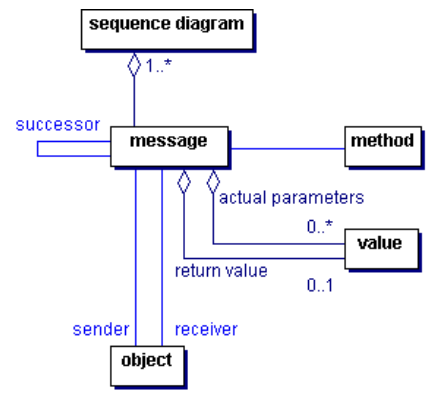

Figure 5. Scenario diagram metamodel. 
and contains only one advice: it notifies the current listener before and after the startingPoint () pointcut is encountered. In case of testsuites, this pointcut is useful for defining a testcase's toplevel methods. This pointcut, along with pointcuts for the events that need to be traced and the type of listener, are all abstract and defined in specific tracers for specific cases, so as to provide maximum flexibility.

The prototype currently offers several "stock" tracers that can be extended for specific cases, e.g., SimpleTracer for capturing basic method and constructor calls and objectTracer for doing so while distinguishing between objects. As an example, consider the occasion in which one merely wants to capture all method and constructor calls. In this case, the SimpleTracer must be extended in a custom tracer that contains definitions for the abstract pointcuts (see Listing 1).

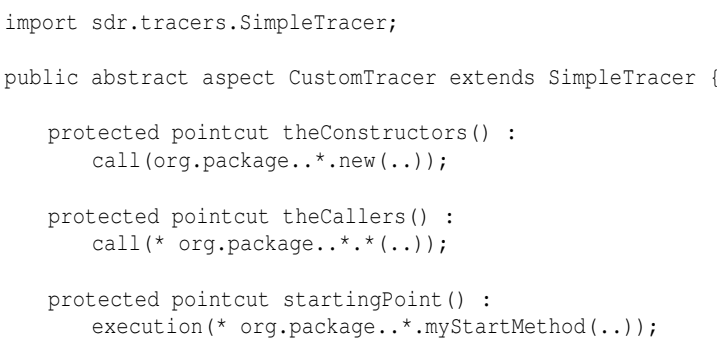

\section{Listing 1. Extending an existing tracer to capture method and constructor calls.}

Listening to events Attached to each tracer is an event listener. In event listeners, one can define how to process the captured events. They implement the EventListener interface and, as such, define routines for starting and stopping the listening phase, and processing generic events.

Our prototype features listeners that perform various tasks, among which are simply printing all events, writing tracefiles, and gathering statistics. Selecting a certain event listener is done by extending the tracer containing the pointcuts and specifying the listener of choice.

Handling testcases For the purpose of tracing testcases, our framework includes a tracer, PerStartTracer, that distinguishes between toplevel methods, i.e., the testcases in a testsuite. It is an extension to the object Tracer and, as such, identifies unique objects. It is extended by a custom tracer, in which the startingPoint () is typically defined as a pattern that matches testclasses that have "Test" in their names. This way, the listener will be informed whenever a (new) testcase in a testclass is executed.

The Sequencelistener is responsible for turning trace events into scenario diagrams. It keeps track of all method and constructor calls and performs the mapping between the trace metamodel and the scenario diagram metamodel. The output consists of a collection of scenario diagrams: one diagram for each of the toplevel methods in the testcases that are being executed.

\section{The JPacman Case Study}

The objective of the JPACMAN case study is to validate our approach, i.e., to verify the usefulness of visualizing testcases, which abstractions are needed, and under which conditions these are to be applied.

We will try to find answers to such questions by studying the JPACMAN application and its testsuite. It is a fairly simple Java (1.5) application consisting of approximately 20 classes and 3000 lines of code, which implements a rudimentary version of the well known Pacman game. JPACMAN is used for teaching purposes in a software testing course at Delft University of Technology. In their lab work, students have to extend both the testsuite and the functionality of JPACMAN using a test-driven / test-first approach, and making use of such tools as Eclipse, Ant, and the code coverage tool CLOVER ${ }^{2}$.

JPACMAN features a JUNIT testsuite of approximately 1000 lines of code. The testcases have been derived systematically using various strategies mostly obtained from Binder [7], including branch coverage (100\%), exercising domain boundaries at on points as well as off points, testing class diagram multiplicities, deriving tests from decision tables (describing, e.g., whether moves on the board are possible), and state and transition coverage (in the state machine describing the overall game behavior). The testsuite consists of 60 testcases in total, divided over 15 high level acceptance tests exercising the top level public interface only, as well as 45 class level unit tests, all implemented in JUNIT (version 4.1).

We have investigated the role that scenario diagrams recovered from these testcases can play when implementing two change requests into JPACMAN. The first is adding an undo feature to JPACMAN, so that users can get back to life if they missed a food element or unintendedly bumped into a monster. Relevant testcases include moving a player or a monster, as well as losing a game. The second change request is turning JPACMAN into a multi-level game including a stack of mazes growing in complexity. Relevant testcases here deal with winning and restarting the game with a different board.

We used SDR to derive scenario diagrams from all 60 testcases, and analyzed them in light of the above change scenarios. ${ }^{3}$ Below we describe our findings, formulate a number of observations, and establish a connection between the scenario diagrams and program comprehension.

\subsection{Unit Tests}

Dynamically obtained key statistics for the JPACMAN case study are shown in Table 1, including such data as the number of constructors, objects and methods, as well as the maximum and most common stack depth.

The data presented was derived from the actual tests themselves, e.g., it does not include the setup phase, in which the

\footnotetext{
2 http: //www. cenqua.com

${ }^{3} \mathrm{~A}$ selection of these diagrams has been included in the most recent JPACMAN distribution.
} 


\begin{tabular}{l|rrrr|rrrr} 
& \multicolumn{4}{|c}{ Unit Tests } & \multicolumn{4}{c}{ Acceptance Tests } \\
\hline & Min & Max & Median & 0.75 -Perc. & Min & Max & Median & 0.75 -Perc. \\
\hline \# Constructors & 0 & 22 & 1 & 1 & 0 & 543 & 1 & 3 \\
\# Methods & 2 & 177 & 13 & 43 & 55 & 1,791 & 73 & 170 \\
\# Objects & 1 & 32 & 4 & 8 & 39 & 578 & 13 & 14 \\
Max. depth & 1 & 7 & 4 & 5 & 7 & 9 & 8 & 8 \\
Most common depth & 1 & 5 & 1 & 3 & 3 & 6 & 5 & 6
\end{tabular}

Table 1. Key statistics for the JPACMAN case study.

fixture is created in which the method under test can be invoked. For that reason, the number of constructors is much lower than the amount of objects involved.

A first observation from this table is that most unit tests are fairly simple in terms of the number of objects and method calls. Half of the unit testcases use four or less objects (median 4), and three quarter of the testcases use fewer than 8 objects (0.75-percentile). Likewise, three quarters of the testcases have fewer than 43 method calls.

Observation 1. Without any abstractions, three quarters of the unit testcases result in scenario diagrams that are small enough to be comprehensible.

A typical example of such a small diagram is shown in Figure 6. The diagram shows how the test method first occupies a given cell, and then verifies that the result is as desired, i.e., that the inhabitant of the cell is indeed equal to the food item that was placed there. By studying this diagram (and those of the related testcases), one can learn to understand how moves are implemented (i.e., by means of double dispatch), making it an excellent basis for implementing an undo functionality.

A more complex example, that just falls within the 0.75 percentile, is shown in Figure 7. Although the diagram is full, it is still useful for one of our change requests, namely adding undo functionality. The diagram at hand illustrates a basic player move, which involves creating a Move object that is linked to the source and target cells, followed by the invocation of the apply () method to actually conduct the move (here, the Command design pattern is used [12]).

While Figure 7 is understandable in itself, it also demonstrates several opportunities for applying abstractions:

- The diagram contains several simple getters, which just result in an self-arrow to the currently active lifeline. Since these do not illustrate any object-interaction (un-

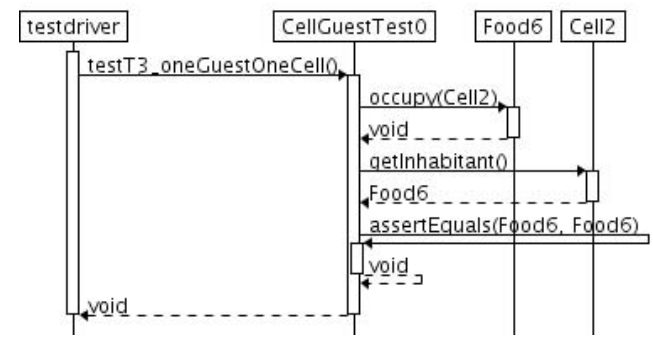

Figure 6. Sequence diagram for a typical unit test. less they result in nested calls), these can usually be safely omitted.

- The diagram contains several recurrent patterns. For example, there are four fairly large blocks in which the first three objects interact in order to determine the current state of the game (which may affect whether the move is allowed at all). All four blocks contain the same sequence of calls and interactions, and there is no need to unfold them all.

- The diagram includes the interactions for conducting the move, as well as for asserting that the move was indeed applied successfully. In understanding how moves are implemented, there is no need for the detailed interactions involved in checking the results. Omitting all interactions obtained from JUNIT's assert methods results in leaving out (or summarizing) two of the repeated blocks mentioned above.

Observation 2. The abstractions "Omit Getters", "Collapse Recurrent Patterns", and "Omit Asserts" can be applied without loss of essential information required for change requests.

With these abstractions in place, the scenario diagrams for most JPACMAN unit tests become sufficiently small. While this will not be the case for all unit testcases in general, we expect a similar result to hold for other systems:

Observation 3. The abstractions in Observation 2 are sufficient to make $90 \%$ of the scenario diagrams of unit testcases understandable.

A final observation that should be made concerning the unit tests, is that according to the XP and test-driven methodologies, tests should run instantaneously. That is, it should be possible to run the full testsuite within several seconds at most, in order not to discourage developers from running the tests continuously. Performance, scalability, system, or acceptance tests that take longer should not be included in the unit testsuite, but in a different suite that should be run at regular intervals (one or more times per day) automatically on the basis of a checkout from the revision system.

As a result, we actually expect such metrics as the number of objects and methods involved in unit tests to be low. In the JPACMAN case, we encountered several testcases in which these metrics were unexpectedly high. The reason turned out to be an error in the loading of maps: the default map (inherited from a superclass of this testcase) was always loaded first, 


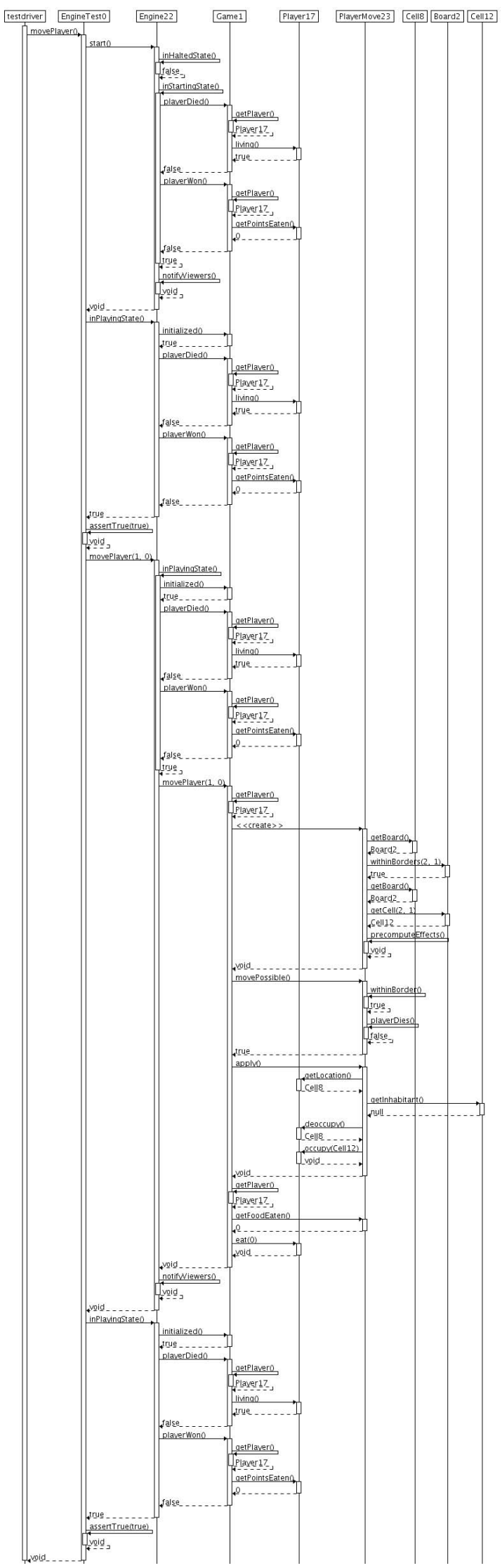

Figure 7. Sequence diagram for a player move.

\begin{tabular}{l|r|r|r|r|r|r|r|r|r} 
Stack depth & 1 & 2 & 3 & 4 & 5 & 6 & 7 & 8 & 9 \\
\hline Frequency & 13 & 29 & 105 & 210 & 614 & 1035 & 245 & 88 & 4
\end{tabular}

Table 2. Depth frequencies in one of the acceptance tests.

and then immediately overwritten by a custom map. Since the default maze was large, and the custom map was deliberately chosen to be small for testing purposes, this was easily seen in the metrics, i.e., the number of constructors involved. While the code was exercised by the testcases, the unnecessary loading was never observed, so no failure was generated.

Observation 4. Dynamically obtained testcase metrics can help to identify faults and refactorings.

\subsection{Acceptance Testing}

When looking at acceptance tests, obviously the figures are substantially larger than those of the unit tests. This is due to the many interactions taking place, and the fact that these tests require more elaborate fixtures, i.e., more objects. This is illustrated by the rightmost portion of Table 1 .

However, the figures for the tests would have been far greater had the test stages not been separated. This holds true especially for the acceptance tests, as in JPACMAN these require the construction of large fixtures, e.g., a map with certain dimensions.

Observation 5. Separation of the testcase stages leads to more readable diagrams without loss of essential information.

By filtering out these phases, we get a smaller diagram enabling us to fully concentrate on the testcase that is being executed. In case the viewer is interested in the initialization or the assertions, it can be viewed in a separate diagram.

Observation 6. In the light of Observation 5, half of the acceptance tests lead to readable scenario diagrams without use of abstractions.

Among the 15 acceptance tests is a relatively large testcase (543 constructors and 1,791 methods) that involves several consecutive movements and game restarts, making it the most complex testcase by far. If we look closer at the metrics for this testcase - the stack depth frequencies in particular we get the figures of Table 2 .

Based on these metrics and an initial viewing, we applied two abstractions: hiding the control flow of constructors, and limiting the stack depth to 2 (Figure 8). This diagram effectively depicts the essence of the testcase at hand: instantiating the Pacman-class (which would normally induce a large amount of interactions), starting the Engine, and conducting the moves. For a more detailed view, one can set the maximum stack depth to 3 , resulting in a diagram that (according to Table 2) features approximately 100 more calls. It is from these detailed diagrams that the necessary information can be 


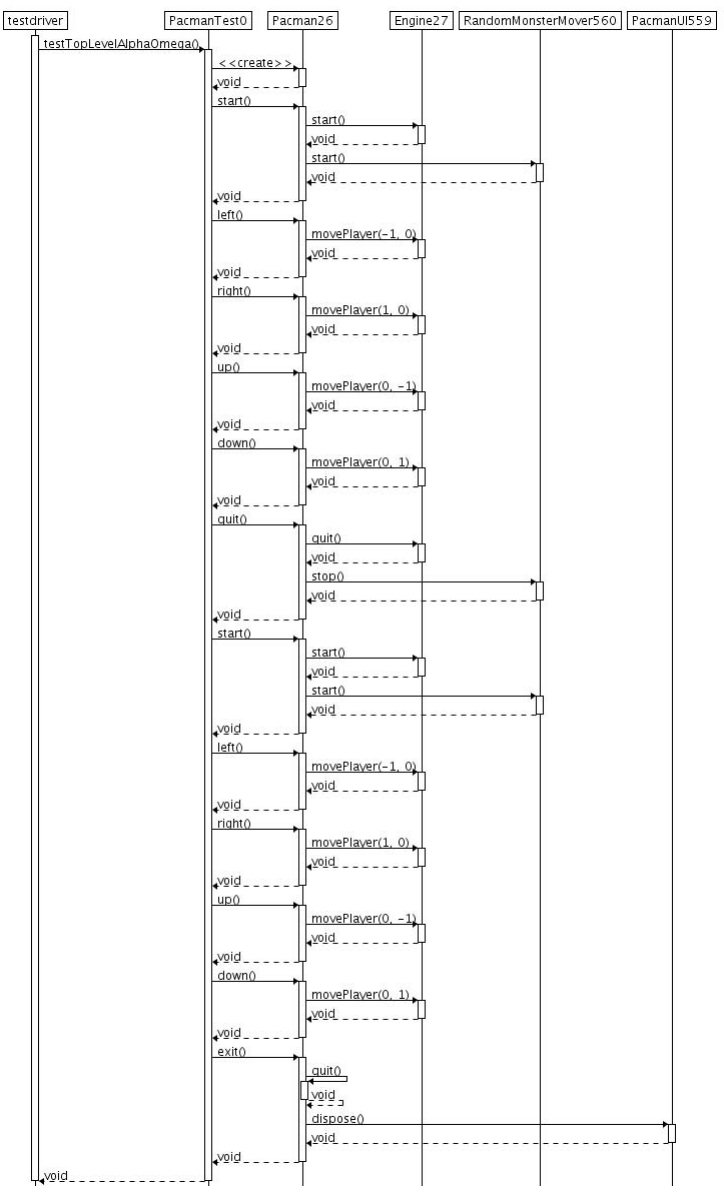

Figure 8. Scenario diagram with a max. stack depth of 2.

derived for gaining insight into the procedure that concerns loading maps: using this knowledge, the change request regarding multiple maps can be facilitated.

Observation 7. Using more advanced abstractions such as stack depth limitation and hiding control flows of constructors, all acceptance tests lead to readable scenario diagrams.

\subsection{Discussion}

The JPACMAN case has taught us several lessons.

First of all, the dynamically obtained scenario diagrams contain lots of detail, such as runtime parameters, actual return values, and occurrences of late binding. While this is useful, especially for change requests that require detailed knowledge, we often get diagrams that are too large to comprehend. This holds true especially in the case of acceptance tests, making abstractions essential in this context.

Separating the testcase stages was clearly a useful measure, as most scenario diagrams became significantly smaller when leaving phases (e.g., the setup) to distinct diagrams.

Several abstractions have been succesfully applied in view- ing the diagrams obtained from the JPACMAN testsuite. Hiding constructors, omitting assertions and stack depth limitation have proven useful to shrink the diagrams without losing the essence of the testcases at hand. The viewer is constantly in control of these abstractions and, by changing the diagram's specifications, can always adjust the abstraction parameters and regenerate the diagram.

With many of the proposed abstraction techniques, however, it is preferable to use them in an interactive fashion, i.e., apply them on-demand. One could think of making messages and objects clickable, and subject to (un-)folding. Using metrics to automatically apply abstractions is a good starting point, but the viewer must ultimately be in control of which information to show and which to hide.

Each testcase that we have treated in our study was a review, resulting in improvements in both our technique and the testcases at hand. Various mistakes (such as duplicate setups) were exposed and have been resolved in the next version.

Threats to validity The case study that we conducted has some limitations that hinder the generalization of any conclusions. First, in spite of its extensive testsuite, JPACMAN is a relatively small system. While certain conclusions and hypotheses can be drawn from the results, they may not hold for large cases: certain abstractions mentioned in this paper may turn out to be insufficient. Second, JPACMAN is an educational example: it is a well-maintained system with a testsuite that covers $100 \%$ of the system's code. One must acknowledge that this is not necessarily true for real life cases, e.g., industrial systems.

\section{Related work}

Using dynamic analysis for program comprehension purposes is an active field of research. This section provides a short overview of related work.

General approaches Various approaches reconstruct scenario and interaction diagrams based on static analysis of program code $[25,16,26,17]$. The techniques that are used vary from mapping of control flow graphs [26] to interprocedural dataflow analysis [25]. A comparison of various approaches is presented in [17].

Sequence diagrams UML sequence diagrams (and variations thereof) have since long been used to visualize dynamically obtained information from a system. We now provide a selection of the existing research in this area.

De Pauw et al. propose several abstractions in reconstructing sequence diagrams [22]. Among these techniques is pattern recognition, and they introduce the concept of execution pattern notation [23]. Their techniques (formerly implemented in Jinsight) are used in a plugin that is part of Eclipse's Test \& Performance Tools Platform (TPTP) Project [3].

Systä et al. aid in the understanding of Java systems in an environment called Shimba, which uses both static analysis and dynamic information [31]. The Shimba environment 
considers static and dynamic information to be complementary and uses static information to bring focus to dynamically generated diagrams, and vice versa.

Jerding et al. have developed the ISVis (Interactive Scenario Visualizer) environment [15]. Its main purpose is to alleviate the architecture localization problem, or the problem of finding the exact location in a system's architecture where a specific enhancement can be inserted. Most of the abstraction techniques that are being used are not fully automatic.

Briand et al. [8] reverse engineer UML sequence diagrams for (1) program understanding in the face of incomplete documentation, and (2) quality assurance, when reverse engineered diagrams are compared with existing design sequence diagrams. Their major contribution lies in the fact that they are among the first to tackle the reverse engineering of UML sequence diagrams from distributed (Java) systems. However, they do not reason from the viewpoint of testsuites, and abstractions play a minor role.

General visualizations Riva et al. [24] combine static and dynamic analysis to reconstruct message sequence charts. In their trace-based approach, they provide an abstraction mechanism based on the decomposition hierarchy that is extracted from the system's source code. It is not described how the scenarios are defined, and in dealing with large diagrams, they only offer manual abstraction techniques.

Furthermore, Pacione et al. make a comparison of dynamic visualization techniques [21] and Hamou-Lhadj et al. discuss trace visualization tools [14].

\section{Conclusions and Future Work}

Testing is an essential part of the software lifecycle. Not only are tests important to guarantee correctness: in the context of Agile methods and eXtreme programming, testcases are used as documentation as well. Moreover, by understanding a system's testsuite, one can gain a great deal of knowledge about its inner workings. It is for this reason that visualizing both units tests and acceptance tests can be of great help in program comprehension.

To this end, we have employed dynamic analysis and scenario diagrams. By gathering knowledge of a system's testsuite, we gain more insight into the way the application works. We have discussed the issues and design choices that we encountered and, through several examples, elaborated on our choices for these techniques.

To compensate for scalability issues associated with dynamic analysis and scenario diagrams, we have established a set of metrics that recommend a number of abstractions that should be used to keep the scenario diagram (for each testcase stage) readable, while preserving the desired amount of detail.

We performed a case study in which we sought to answer the research questions that were posed in the introduction. Based on our experiences with this case study, we can now formulate answers to these questions:
- JUNIT tests are a useful starting point for program comprehension. They induce scenarios that effectively decompose a system's functionalities.

- The scenario diagrams that are obtained from these tests offer knowledge about a system's inner workings. Our case study has shown that through the application of several abstractions, scenario diagrams effectively visualize how testcases (and the associated functionalities) work, which aids greatly in planning change requests.

- The amount and the type of abstractions that are necessary to make the scenario diagram of a testcase sufficiently understandable, depend greatly on the type of tests. We have listed several abstraction techniques and implemented part of them in our case study.

- Our experiences indicate that in case of unit tests, simple techniques such as omission of getters and setters and collapsing recurrent patterns suffice, whereas more complex acceptance tests require more complicated filterings such as stack depth limitation and control flow hiding.

Having answered our research questions, we now conclude this section by listing our contributions:

- We have presented a technique to effectively trace the various stages of a JUNIT testcase.

- We have listed a broad range of abstractions in the context of scenario diagrams, some existing and some new, of which some were implemented in a prototype tool. These were automatically applied, based on metrics that were collected during execution.

- By means of an extensive case study, we have shown the extent to which our techniques are useful for testsuite and program understanding.

Future work Developing visualizations that are specifically optimized for testsuite comprehension is a relatively new field of research. Besides investigating existing general purpose visualizations, we have established a number of research directions that we wish to pursue in the future.

Among others, we plan to examine new abstraction techniques that help make test-scenario diagrams easier to understand. Currently, we have a preliminary list of such abstractions (see Section 3.2), but further research on a set of diverse case studies remains necessary to determine the adequacy of these abstractions in the context of testing.

The JPACMAN case yielded interesting results, but focusing on a more complex system will undoubtedly raise new issues. Among the issues that we expect are: (1) how to deal with threads, (2) how to handle incomplete testsuites, (3) how to deal with software systems that have no clear unit testing approach and rely merely on integration and acceptance tests (e.g., Apache Ant [13]).

Finally, we feel that that we should integrate our tool within the Eclipse framework, which would allow for a more interactive approach to gathering knowledge about the testsuite and the complete system. Moreover, integration into Eclipse will 
make the tool more attractive to a broader audience.

Acknowledgments Part of the research described in this paper was sponsored by NWO via the Jacquard Reconstructor project and by the ESI TRADER project.

\section{References}

[1] AspectJ: The AspectJ project at Eclipse.org, http://www . eclipse.org/aspect j/.

[2] InsectJ: A generic instrumentation framework for collecting dynamic information within Eclipse, http://insectj. sourceforge.net/.

[3] The Eclipse Test \& Performance Tools Platform (TPTP) Project, http://www.eclipse.org/tptp/.

[4] K. Beck. Extreme Programming Explained - Embrace Change. Addison-Wesley, 1999.

[5] K. Beck. Test-Driven Development: By Example. AddisonWesley, 2003.

[6] K. Beck and E. Gamma. Test infected: Programmers love writing tests. Java Report, 3(7):51-56, 1998.

[7] R. Binder. Testing Object-Oriented Systems: Models, Patterns, and Tools. Addison-Wesley, 2000.

[8] L.C. Briand, Y. Labiche, and J. Leduc. Toward the reverse engineering of uml sequence diagrams for distributed java software. IEEE Transactions on Software Engineering, 32(9):642-663, 2006.

[9] S. Demeyer, S. Ducasse, and O. Nierstrasz. Object Oriented Reengineering Patterns. Morgan Kaufmann, 2002.

[10] A. van Deursen. Program comprehension risks and benefits in extreme programming. In Proc. 8th Working Conf. on Reverse Engineering (WCRE'01), pages 176-185, 2001.

[11] A. Forward and T. C. Lethbridge. The relevance of software documentation, tools and technologies: a survey. In Proc. ACM symposium on Document engineering (DocEng'02), pages 2633, 2002.

[12] E. Gamma, R. Helm, R. Johnson, and J. Vlissides. Design Patterns Elements of Reusable Object-Oriented Software. Addison-Wesley, 1994.

[13] J. van Geet and A. Zaidman. A lightweight approach to determining the adequacy of tests as documentation. In Proc. 2nd Int. Workshop on Program Comprehension through Dynamic Analysis (PCODA'06), pages 21-26, 2006.

[14] A. Hamou-Lhadj and T.C. Lethbridge. A survey of trace exploration tools and techniques. In Proc. 2004 Conf. of the Centre for Advanced Studies on Collaborative Research (CASCON'04), pages 42-55, 2004.

[15] D. Jerding and S. Rugaber. Using visualization for architectural localization and extraction. In Proc. 4th Working Conf. on Reverse Engineering (WCRE'97), page 56, 1997.

[16] R. Kollmann and M. Gogolla. Capturing dynamic program behaviour with UML collaboration diagrams. In Proc. 5th Conf. on Software Maintenance and Reengineering (CSMR'01), pages 58-67, 2001.

[17] R. Kollmann, P. Selonen, E. Stroulia, T. Systä, and A. Zündorf. A study on the current state of the art in tool-supported UMLbased static reverse engineering. In Proc. 9th Working Conf. on Reverse Engineering (WCRE'02), pages 22-32, 2002.

[18] B. Marick. Agile methods and agile testing. http://testing.com/agile/agile-testing-essay.html (accessed October 9th, 2006), 2004.

[19] K. W. Miller. Test driven development on the cheap: text files and explicit scaffolding. J. Comput. Small Coll., 20(2):181$189,2004$.

[20] OMG. UML 2.0 infrastructure specification. Object Management Group, http://www.omg.org/, 2003.

[21] M.J. Pacione, M. Roper, and M. Wood. Comparative evaluation of dynamic visualisation tools. In Proc. 10th Working Conf. on Reverse Engineering (WCRE'03), pages 80-89, 2003.

[22] W. De Pauw, E. Jensen, N. Mitchell, G. Sevitsky, J. Vlissides, and J. Yang. Visualizing the Execution of Java Programs, pages 151-162. Springer-Verlag, 2001.

[23] W. De Pauw, D. Lorenz, J. Vlissides, and M. Wegman. Execution patterns in object-oriented visualization. In Proc. 4th USENIX Conf. on Object-Oriented Technologies and Systems (COOTS'98), pages 219-234, 1998.

[24] C. Riva and J. V. Rodriguez. Combining static and dynamic views for architecture reconstruction. In Proc. 6th Conf. on Software Maintenance and Reengineering (CSMR'02), pages 47-55, 2002.

[25] A. Rountev and B. H. Connell. Object naming analysis for reverse-engineered sequence diagrams. In Proc. 27th Int. Conf. on Software Engineering (ICSE'05), pages 254-263, 2005.

[26] A. Rountev, O. Volgin, and M. Reddoch. Static control-flow analysis for reverse engineering of UML sequence diagrams. In Proc. 6th Workshop on Program Analysis for Software Tools and Engineering (PASTE'05), pages 96-102, 2005.

[27] J. Rumbaugh, I. Jacobson, and G. Booch. The Unified Modeling Language Reference Manual. Addison-Wesley, 1998.

[28] K. Schwaber and M. Beedle. Agile Software Development with Scrum. Prentice Hall, 2001.

[29] R. Sharp and A. Rountev. Interactive exploration of UML sequence diagrams. In Proc. 3rd Int. Workshop on Visualizing Software for Understanding and Analysis (VISSOFT'05), pages 8-15, 2005.

[30] J. Stapleton. Dynamic Systems Development Method: The method in practice. Addison-Wesley, 1997.

[31] T. Systä, K. Koskimies, and H. Müller. Shimba - an environment for reverse engineering Java software systems. Software - Practice and Experience, 31(4):371-394, 2001.

[32] S. Yang, M. M. Burnett, E. DeKoven, and M. Zloof. Representation design benchmarks: a design-time aid for vpl navigable static representations. J. Visual Lang. \& Computing, 8(56):563-599, 1997.

[33] A. Zaidman. Scalability Solutions for Program Comprehension through Dynamic Analysis. PhD thesis, University of Antwerp, 2006.

[34] A. Zaidman, T. Calders, S. Demeyer, and J. Paredaens. Applying webmining techniques to execution traces to support the program comprehension process. In Proc. 9th Conf. on Software Maintenance and Reengineering (CSMR'05), pages 134142, 2005. 

TUD-SERG-2006-003 ISSN 1872-5392 